International Journal of Pure and Applied Mathematics

Volume 105 No. 3 2015, 523-528

ISSN: 1311-8080 (printed version); ISSN: 1314-3395 (on-line version)

url: http://www.ijpam.eu

doi: http://dx.doi.org/10.12732/ijpam.v105i3.17

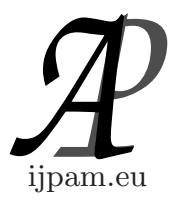

\title{
A LYAPUNOV APPROACH IN THE DERIVATIVE APPROXIMATION USING A DYNAMIC SYSTEM
}

\author{
Paolo Mercorelli \\ Institute of Product and Process Innovation \\ Leuphana University of Lueneburg \\ Volgershall 1, D-21339 Lueneburg, GERMANY
}

\begin{abstract}
This paper deals with an approximation of a derivative using a dynamic system of the first order. After formulating the problem, two propositions, allowing to find a possible approximation consisting of a dynamic system, are proven. In particular, a proposition based on a Lyapunov approach is proven to show the convergence of the approximation. The second proposition is a constructive one and shows directly the dynamic approximation.
\end{abstract}

AMS Subject Classification: 26A24, 93C55, 49K15

Key Words: derivatives, discrete-time systems, computational methods

\section{Introduction and Motivation}

Derivatives and structures using derivatives, as for instance PD- controllers, are very often used in industrial applications. Such kind of controllers are much used in practical applications of any nature. They are used also in electromagnetic valve control, [1] and more recently in [2], [3], [4] and [5]. This contribution

Received: October 24, 2015

(c) 2015 Academic Publications, Ltd. url: www.acadpubl.eu 
emphasizes some mathematical aspects of the algorithm which the author used in practical applications such as for instance in [6] and in [7]. In particular, in [6] this algorithm is used in designing a velocity observer in the context of a throttle valve control. In [7] a similar algorithm is used to estimate the parameters identification in the application in which a synchronous motor is proposed. This paper extends the results presented in [8], removing some conservative hypotheses, and proposes an approximated derivative structure to be taken into account for such kind of applications so that spikes, noise and any other kind of undesired signals which occur from the derivatives can be reduced. After the problem formulation this paper proves a proposition which allows to build this possible approximation of the derivative using a dynamic system. The paper is structured as follows. In Section 2 the problem formulation and a possible solution are proposed. At the end a remark discusses the results and the conclusion closes the paper.

\section{An Approximated Derivative Structure}

Using the derivative structures, imprecision occurs. The imprecision is due to spikes generating power dissipation. The idea is to find an approximated structure of general derivatives as they occur in mathematical calculations which are often used also in technical problems as proportional derivative controllers. The following formulation states the problem in a mathematical way.

Problem 1. If the following differential expression is given:

$$
r(t)=k_{d} \frac{d e_{y}(t)}{d t}
$$

where $e_{y}(t)=y_{d}(t)-y(t)$, and $y_{d}(t)$ and $y(t)$ are the desired and achieved position trajectories, respectively. In particular, $t$ represents the time variable with $t \in \mathbb{R}, y_{d}(t)$ and $y(t) \in \mathbb{R}$. The aim of the proposed approach is to look for an approximating expression $\hat{r}\left(t, e_{y}(t), k_{d}, k_{a p p}\right)$, where $k_{d}$ is a constant and $k_{a p p}$ is a parameter, such that:

$$
\lim _{k_{a p p} \rightarrow+\infty} r(t)-\hat{r}(t)=0 .
$$

Proposition 1. Considering Eq. (1), then there exists a function $\mathcal{M}>0$ such that, if the following dynamic system is considered:

$$
\frac{d \hat{r}(t)}{d t}=-\mathcal{M}\left(\hat{r}(t)-k_{d} \frac{d e_{y}(t)}{d t}\right),
$$


where $r(t)$ represents the real derivative function and $\hat{r}(t)$ the approximated derivative function, considering the following error:

$$
e_{r}(t)=r(t)-\hat{r}(t)
$$

if

$$
\frac{\partial \hat{r}(t)}{\partial t}>\frac{\partial r(t)}{\partial t} \forall t
$$

and

$$
\mathcal{M}>0 \quad \forall t
$$

then

$$
\lim _{t \rightarrow+\infty} e_{r}(t)=0
$$

Proof. Considering the following approximate dynamic system:

$$
\frac{d \hat{r}(t)}{d t}=-\mathcal{M}\left(\hat{r}(t)-k_{d} \frac{d e_{y}(t)}{d t}\right),
$$

where $\mathcal{M}$ can be a function of $e_{y}(t)$ or a parameter with $\mathcal{M} \in \mathbb{R}$. If

$$
e_{r}(t)=r(t)-\hat{r}(t)
$$

then

$$
\frac{d e_{r}(t)}{d t}=\frac{d r(t)}{d t}-\frac{d \hat{r}(t)}{d t}
$$

After inserting Eq. (8) into Eq. (10) it follows:

$$
\frac{\partial e_{r}(t)}{\partial t}=\frac{\partial r(t)}{\partial t}+\mathcal{M}\left(\hat{r}(t)-k_{d} \frac{d e_{y}(t)}{d t}\right)
$$

if Eq. (1) is taken into consideration, then Eq. (11) becomes as follows:

$$
\frac{\partial e_{r}(t)}{\partial t}+\mathcal{M} e_{r}(t)=\frac{\partial r(t)}{\partial t} .
$$

If the following Lyapunov function is considered:

$$
V\left(e_{r}(t)\right)=\frac{1}{2} e_{r}^{2}(t),
$$

and considering that:

$$
\frac{\partial V\left(e_{r}(t)\right)}{\partial t}=e_{r}(t) \frac{\partial e_{r}(t)}{\partial t} .
$$


According to Eq. (12) it is possible to write the following expression:

$$
e_{r}(t)=\frac{\frac{\partial r(t)}{\partial t}-\frac{\partial e_{r}(t)}{\partial t}}{\mathcal{M}},
$$

and thus from Eq. (14) it follows that:

$$
\frac{\partial V\left(e_{r}(t)\right)}{\partial t}=\frac{\frac{\partial e_{r}(t)}{\partial t} \frac{\partial r(t)}{\partial t}-\left(\frac{\partial e_{r}(t)}{\partial t}\right)^{2}}{\mathcal{M}} .
$$

Considering Eq. (8), then:

$$
\frac{\partial e_{r}(t)}{\partial t} \frac{\partial v(t)}{\partial t}=\left(\frac{\partial r(t)}{\partial t}\right)^{2}-\frac{\partial \hat{r}(t)}{\partial t} \frac{\partial r(t)}{\partial t},
$$

and

$$
\frac{\partial e_{r}(t)}{\partial t} \frac{\partial r(t)}{\partial t} \leq-\frac{\partial \hat{r}(t)}{\partial t} \frac{\partial r(t)}{\partial t},
$$

if

$$
\frac{\partial \hat{r}(t)}{\partial t}>\frac{\partial r(t)}{\partial t} \quad \forall t
$$

as stated by the hypothesis in Eq. (5), then:

$$
\frac{\partial \hat{r}(t)}{\partial t} \frac{\partial r(t)}{\partial t}>\left(\frac{\partial r(t)}{\partial t}\right)^{2} \forall t
$$

and thus

$$
\frac{\partial \hat{r}(t)}{\partial t} \frac{\partial r(t)}{\partial t}>0 \quad \forall t
$$

Considering that:

$$
\mathcal{M}>0 \quad \forall t
$$

as stated by the hypothesis in Eq. (6), then:

$$
\frac{\partial V\left(e_{r}(t)\right)}{\partial t}<0 \quad \forall t
$$

So Eq. (12) is uniformly asymptotically stable and Eq. (7) is proven.

Proposition 2. The dynamic system

$$
\begin{aligned}
\frac{d \eta(t)}{d t} & =-\frac{k_{a p p}}{k_{d}} \eta(t)+\frac{k_{a p p}}{k_{d}}\left(k_{p} e_{y}(t)-k_{a p p} e_{y}(t)\right) \\
\hat{r}(t) & =\eta(t)+k_{a p p} e_{y}(t),
\end{aligned}
$$

where function $\eta(t) \in \mathbb{R}$, solves the problem defined above. 
A supplementary variable is defined as:

$$
\eta(t)=\hat{r}(t)-\mathcal{N}\left(e_{y}(t)\right)
$$

where $\mathcal{N}\left(e_{y}(t)\right)$ is a function to be designed with $\mathcal{N}\left(e_{y}(t)\right) \in \mathbb{R}$.

Considering that:

$$
\frac{d \eta(t)}{d t}=\frac{d \hat{r}(t)}{d t}-\frac{d N\left(e_{y}(t)\right)}{d t}
$$

let

$$
\mathcal{M} k_{d} \frac{d e_{y}(t)}{d t}=\frac{d N\left(e_{y}(t)\right)}{d t}=\frac{d N\left(e_{y}(t)\right)}{d e_{y}(t)} \frac{d e_{y}(t)}{d t} .
$$

If $\mathcal{N}\left(e_{y}(t)\right)=k_{\text {app }} e_{y}(t)$, then $\mathcal{M}=\frac{k_{\text {app }}}{k_{d}}$. If $k_{d}>0$, then the asymptotical stability is always guaranteed for $k_{a p p}>0$ and the rate of convergence can also be specified by $k_{a p p}>0$. From (25),

$$
\hat{r}(t)=\eta(t)+k_{\text {app }} e_{y}(t)
$$

and substituting (28) into (8), it follows (24).

\section{Conclusion}

This paper deals with an approximation of a derivative using a dynamic system to avoid spikes and noise. After formulating the problem, two propositions are proven which allow to find a possible solution.

\section{References}

[1] C. Tai, A. Stubbs, and T. Tsao. Modeling and control design of an electromagnetic engine valve. In Proceedings of the American control conference, volume 4, pages 2890-2895, Arlington, Va, 2001. DOI:10.1109/ACC.2001.946339.

[2] P. Mercorelli. A two-stage augmented extended Kalman filter as an observer for sensorless valve control in camless internal combustion engines. IEEE Transactions on Industrial Electronics, 59(11):4236-4247, 2012. DOI:10.1109/TIE.2012.2192892. 
[3] P. Mercorelli. An anti-saturating adaptive preaction and a slide surface to achieve soft landing control for electromagnetic actuators. IEEE/ASME Transactions on Mechatronics, 17(1):76-85, 2012. DOI:10.1109/TMECH.2010.2089467.

[4] P. Mercorelli. A hysteresis hybrid extended Kalman filter as an observer for sensorless valve control in camless internal combustion engines. IEEE Transactions on Industry Applications, 48(6):1940-1949, 2012. DOI:10.1109/TIA.2012.2226193.

[5] P. Mercorelli. A two-stage sliding-mode high-gain observer to reduce uncertainties and disturbances effects for sensorless control in automotive applications. IEEE Transactions on Industrial Electronics, 62(9):5929-5940, 2015. DOI:10.1109/TIE.2015.2450725.

[6] P. Mercorelli. Robust feedback linearization using an adaptive pd regulator for a sensorless control of a throttle valve. Mechatronics a journal of IFAC. Elsevier publishing, 19(8):1334-1345, 2009. DOI:10.1016/j.mechatronics.2009.08.008.

[7] P. Mercorelli. A decoupling dynamic estimator for online parameters identification of permanent magnet three-phase synchronous motors. In Proc. 16 th IFAC International Symposium on System Identification 2012, pages 757-762, Brussels, 2012. DOI:10.3182/20120711-3-BE-2027.00131.

[8] P. Mercorelli. Derivative approximation using a discrete dynamic system. International Journal of Pure and Applied Mathematics, 85(4):813-817, 2013. DOI: 10.12732/ijpam.v85i4.15. 